\title{
The Mechanism Underlying Heart Rate and Pacemaking Activity Decline in Developing Sinoatrial Node of the Rabbit Heart
}

\author{
Azzah M Alghamdi1 ${ }^{1,2}$, Craig P Testrow ${ }^{1}$, Dominic G Whittaker ${ }^{1,3}$, Henggui Zhang ${ }^{1}$ \\ ${ }^{1}$ University of Manchester, Manchester, UK \\ ${ }^{2}$ King Abdul-Aziz University KAU, Jeddah, Saudi Arabia \\ ${ }^{3}$ University of Leeds, Leeds, UK
}

\begin{abstract}
Diagnosis of heart disease and its treatment are based largely on our understanding of the electrophysiology of adult myocardium. However, the marked difference in the electrical action potential (AP)between neonatal and adult cardiac myocytes suggests a different set of molecular bases in neonatal myocytes, and therefore different treatment for new-borns. In this study we present a new mathematical model of sinoatrial node (SAN) cells of the neonatal rabbit ,by modifying densities or kinetics of $I_{N a}$, $I_{C a L}, I_{f}, I_{K r}, I_{K s}$, and $I_{N a C a}$ in the adult rabbit SAN cell models developed by Zhang et al., based on available experimental data obtained from new-born rabbit SAN cells. The new model reproduced APs similar to experimental recordings from neonatal myocytes, with a faster pacemaking rate. Using the new model, we investigated how age-related changes in ionic currents modulate pacemaking AP morphology, demonstrating the model as a useful tool for testing the effects of drugs on neonatal SAN cells to obtain a better quantitative understanding of differences between neonatal and adult physiology.
\end{abstract}

\section{Introduction}

The function of the heart is compromised during maturation in many species leading to a decrease in the heart rate (HR)[1]. Experimental studies have shown that there are significant developmental changes in the expression and function of ion channels and other cellular elements, leading to a postnatal alternation in the spontaneous pacemaking activity of the transmembrane potential in isolated rabbit SAN cells [2]. This may be responsible for the observed differences in the automaticity and characteristics of the membrane potentials in the adults as compared to the neonatal hearts, including a reduction in spontaneous beating rate, an increase in action potential duration (APD), the intrinsic cycle length(CL), and an increased maximal diastolic potential (MDP)[2].

In this study, we attempted to provide a modelling framework to underpin the ionic mechanism for the developmental maturation of the rabbit SAN at the single cell level based on combined experimental findings on channel properties and gene expression of the ion channel currents and intracellular calcium handling. Using the model we investigated how the remodelling in the expression and function of different ion channels and other intracellular $\mathrm{Ca} 2+$ handling contribute to the alteration of the pacemaking activity of the SAN cells, resulting in the slowing down of the heart rate in the adult heart.

\section{Methods}

Based on the adult rabbit SAN cell model developed by Zhang et al.[3], we modified the formulations of ionic currents and their current densities of the model to incorporate experimental data obtained from neonatal rabbit SAN cells. The main changes made to the Zhang et al. [3] model are summarized as follows.

\subsection{Fast sodium current, $I_{\mathrm{Na}}$}

$I_{\mathrm{Na}}$ was described using a Hodgkin-Huxley formulations, which were incorporated into the Zhang et al. model based on experimental data from Brousticci et al.[4] of both new-born and young rabbit SAN cells. The activation and inactivation steady state curves of the model were fitted to the experimental data of Baruscotti et al.[4], with a shift to the $V_{1 / 2}$ of the activation curve (as seen in Figure1A), considering the experimental data were obtained at room temperature $20^{\circ} \mathrm{C}$ while the model was developed for the body temperature. In addition, $\mathrm{aQ}_{10}$ correction of the current density was also considered accounting for the temperature difference. To validate the model, simulated voltage-clamp data of $I_{\mathrm{Na}}$ were compared to experimental data. In the voltage clamp simulation, $I_{\mathrm{Na}}$ was computed from a holding potential of $-60 \mathrm{mV}$, which 
was followed by a series of test potentials lasting $10 \mathrm{~ms}$, varying from $-60 \mathrm{mV}$ to $45 \mathrm{mV}$ with a $5 \mathrm{mVincrement}$. The maximal channel conductance of the sodium current, $g_{\mathrm{Na}, \max }$, was determined by reproducing the experimental data on the $I-V$ relationship as represented in Figure 1B.Based on the experimental data, the peak $\mathrm{I}_{\mathrm{Na}}$ current density of the neonate $I-V$ curve was greater than that of the young. This resulted in a relatively larger "window current" seen in the neonate case [4]
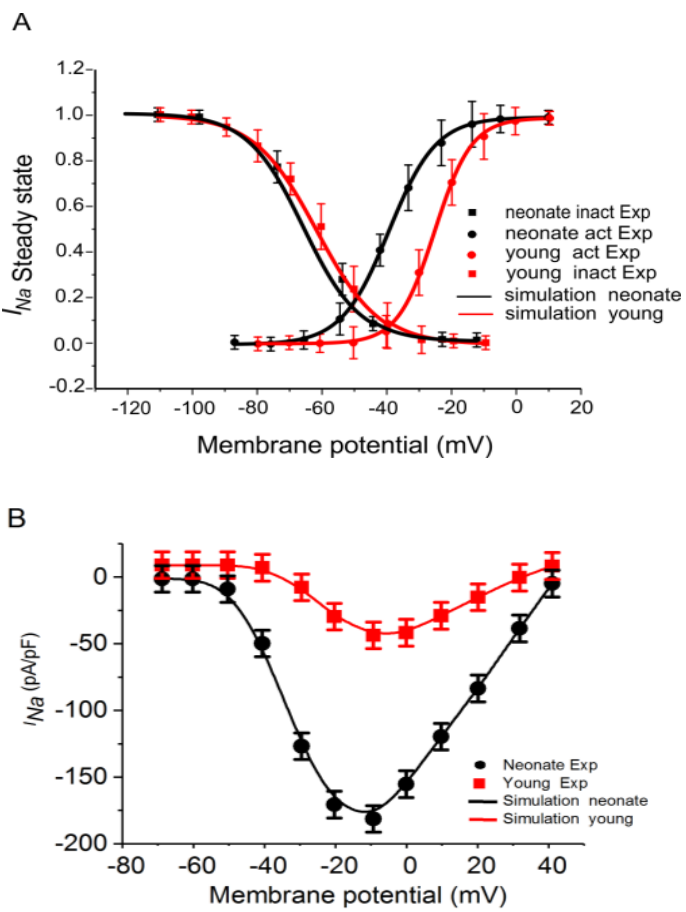

Figure 1.(A) Steady state activation and inactivation curves of the $I_{\mathrm{Na}}$ current in neonate and young age groups. Modelling date fits to experimental data.(B)Simulated $I-V$ relationship between the two age group, which were validated against experimental data from Brousticci et al.[4].

\subsection{L-type calcium current, $I_{\mathrm{Ca}, \mathrm{L}}$}

The $I_{\mathrm{Ca}, \mathrm{L}}$ formulation used in Zhang et al.[3]model was modified based on the available experimental data from Protas et al.[5]for the neonate SAN cells. The steady state activation and inactivation curves were fitted to the experimental data, but with a $5 \mathrm{mV}$ rightward shift to the $\mathrm{V} 1 / 2$ of the inactivation curve and a $5 \mathrm{mV}$ leftward shift to the V1/2 of activation curve in the adult, resulting in an increased $I_{\mathrm{CaL}}$ window current for the adult cells as seen in Figure 2A. Using voltage clamp protocol as used in experiments (with a holding potential of $-50 \mathrm{mV}$, which was followed by a series of test potentials lasting $300 \mathrm{~ms}$, varying from $-50 \mathrm{mV}$ to $50 \mathrm{mV}$ with a $10 \mathrm{mV}$ increment),
$I_{\mathrm{Ca}, \mathrm{L}}$ was computed and the established $I-V$ relationship curve of $I_{\mathrm{Ca}, \mathrm{L}}$ on neonate and adult rabbit SAN cells were validated against experimental data[5] as illustrated in Figure2B.

A

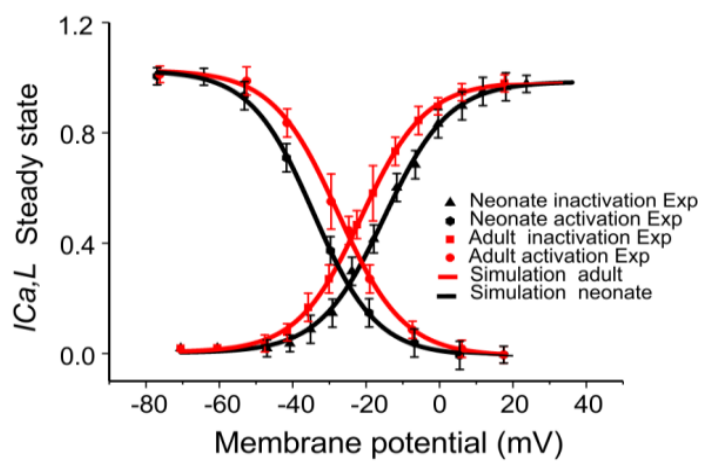

B

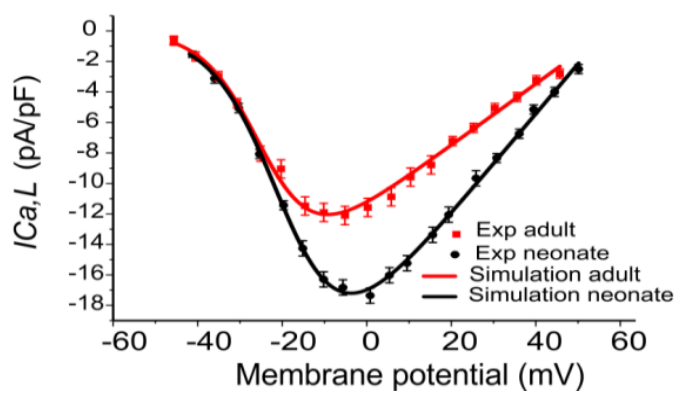

Figure2.(A)Fitted steady state activation and inactivation gate curves of $I_{\mathrm{Ca}, \mathrm{L}}$ in two neonate and adult age groups.(B) Simulated $I-V$ relationship of the two age group, which were validated against experimental data by Protas et al.[5].

\subsection{Funny current, $I_{f}$}

The formulation of $I_{\mathrm{f}}$ in the Zhang et al.[3]model was modified based on the available data by Accil et al.[6]and Yang et al.[7]. The activation curve was fitted to experimental data with an age-related negative shift of the activation $\mathrm{V}_{1 / 2}$ by $7 \mathrm{mv}$ (as shown in Figure $3 \mathrm{~A}$ ). It was also shown that the If current density is greater in the neonate than in the adult SAN cells, with an increase of the maximal conductance of $g_{\mathrm{Na}}, g_{\mathrm{f}, \mathrm{K}}$ by $70 \%$ in the neonate as compared to the adult cells. . The simulated $I-V$ curve (Figure3B) was validated against experiment data by Protas et al.[8] and Honjo et al.[9].

\subsection{Other ion currents}

Other ion channel currents including $I_{\mathrm{Kr}}, I_{\mathrm{Ks}}$, and $I_{\mathrm{NaCa}}$ were modified on the basis of changes in the expression of ion channels and $\mathrm{Ca}^{2+}$-handling proteins during development as a previous quantitative PCR, insitu 
hybridization and immunohistochemistry study investigated[10]. This was done through modification of the maximal conductance of $g_{\mathrm{Kr}}$ by $60 \%, g_{\mathrm{Ks}}$ by $25 \%$ and $K_{\mathrm{NaCa}}$ by $50 \%$ in the Zhang et al. [3] model
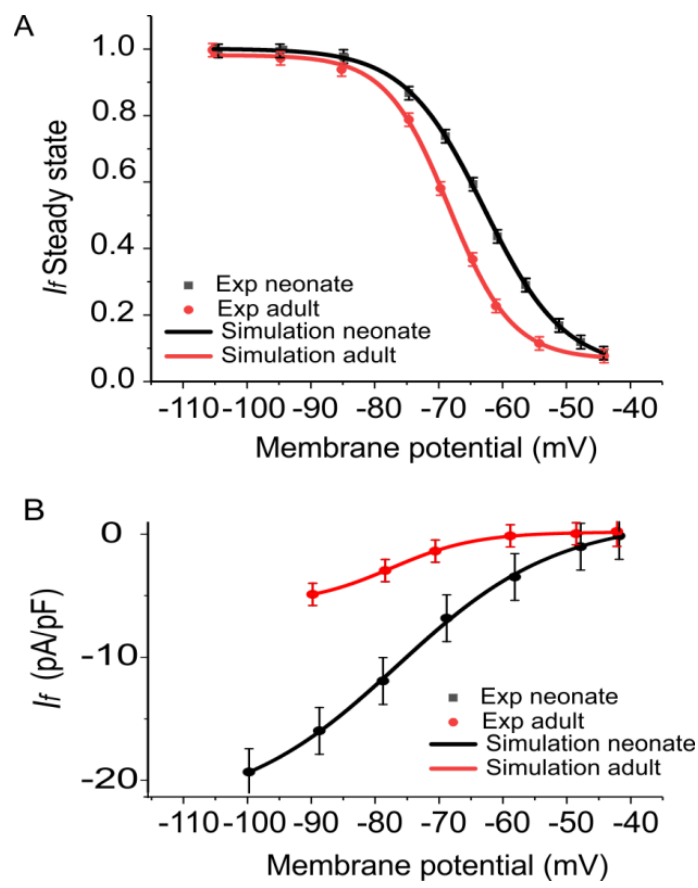

Figure3.(A) Fitted steady state activation curves of the $I_{\mathrm{f}}$ current in the neonate and adult age groups. (B) Simulated $I-V$ relationship, which was validated against experimental data by Protas et al. [8] and Honjo et al. [9].

\section{Results}

Models of the pacemaking action potentials for the neonate and adult SAN cells were developed by incorporating the age-related remodelling of different ion channels $\left(I_{\mathrm{Na}}, I_{\mathrm{Ca}, \mathrm{L}}, I_{\mathrm{f}}, I_{\mathrm{Kr}}\right.$ and $I_{\mathrm{Ks}}$ as well as $\left.I_{\mathrm{NaCA}}\right)$ and calcium handling into the Zhang et al. (3) model as a basal adult SAN single cell model. Figure 4 illustrates the simulated AP of the neonate SAN cell, which is compared to that of the adult. It was shown that the neonate model reproduces APs with their shape and characteristic matching to experimental data. Mainly the neonatal APs have faster spontaneous activities, shorter durations and greater amplitudes as compared to those of the adult. The computed CL (i.e. the time interval between two successive pacemaking APs) of the neonate was $277 \mathrm{~ms}$, which was markedly smaller than that of 327 in CL in the adult, showing an $18 \%$ prolongation of the CL due to age(as shown in Figure4B). This is quantitatively consistent with the experimental observation [10], which showed that the heartbeat of rabbits was slowed down during postnatal development by $15 \%$. Heart rate has been measured as around $230 \pm 50 \mathrm{bpm}$ for neonates [10],[11], which is very close to our simulation data of $217 \mathrm{bpm}$ in neonates and $160 \mathrm{bpm}$ in adults(Figure 5B)respectively.
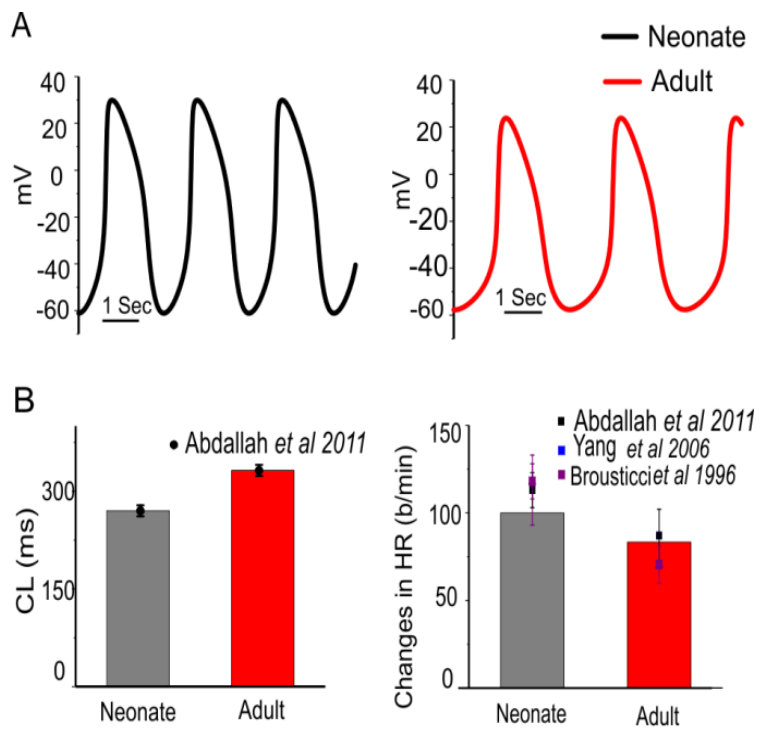

Figure4. (A) Simulated pacemaking action potentials in the neonate (black lines) and the adult (red lines) SAN cells. (B) Comparison of CL (right), HR (left) between the two age groups, which were validated by experimental data.

\section{Discussion}

The simulation result confirmed that there was an agedependent change in the AP characteristics and pacemaking rate, causing a decreased SAN pacemaking activity in the adult. This finding indicates that the fast AP of the neonatal group results from changes in the balance of ionic currents that activate during the diastolic depolarisation phase compared to the adult group. A previous study [4] has reported that $I_{\mathrm{Na}}$ was present in rabbit central SAN cells at birth. The observation of this ion current during development showed that by the 40th postnatal day, the $I_{\mathrm{Na}}$ had fully disappeared. It was found neonate cells of central SA node exhibited a noticeable window current, resulting in the likely higher availability of sodium current in the diastolic depolarisation phase in neonates. With development, the $\mathrm{V}_{1 / 2}$ of the activation curve shifted in the positive direction, while the inactivation remained unaltered, resulting in a reduced overlap of the two curves and hence less window current. This decline in the window currents may help to explain how the principal current disappears gradually with age to become non-existent in adult SAN and be replaced by $I_{\mathrm{Ca}, \mathrm{L}}$.

The $I_{\mathrm{Ca}, \mathrm{L}}$ current was identified as the primary pacemaking current which initiates the APs in the central 
adult SAN cells [2, 3].As this simulation result shows, in the neonate, it has a secondary contribution of automaticity. The greater $I_{\mathrm{Ca}, \mathrm{L}}$ current density in the neonate and the modulation of $I_{\mathrm{Ca}, \mathrm{L}}$ kinetics with age may play an important role in the high peak value of the APs in the neonate[5].

The present study also demonstrated an association between the age-dependent difference in current density and the $\mathrm{V}_{1 / 2}$ of the $I_{\mathrm{f}}$ activation curve, which also contribute to a slowing down of the pacemaking rate in the adult. Previous studies[6][7] demonstrated that the pacemaker current $I_{\mathrm{f}}$, exhibited an age-dependent decrease in current density which was assumed to result from baseline cAMP reduction levels in the $\mathrm{SA}$ node, suggesting that the combined action between $I_{\mathrm{f}}$ channels and fewer cAMP molecules are the main cause of the activation curve shift towards hyperpolarisation in older age groups, leaving fewer $I_{\mathrm{f}}$ channels available to initiate diastolic depolarisation, a key determinant of the heart rate decrease of the SA node.

The contribution of the delayed rectifier $\mathrm{K}^{+}$currents and $\mathrm{Na}^{+} / \mathrm{Ca}^{2+}$ exchange current to SAN pacemaking in adult have been studied[12], but the role of developmental changes in potassium currents in the sinus node remains unexplored. Based on gene expression level measurements, the great abundance of $I_{\mathrm{Kr}}, I_{\mathrm{Ks}}$ and $I_{\mathrm{NaCa}}$ in neonate SAN was of small consequence on the pacemaking effect.

In conclusion, this simulation study provided a novel computational model of the action potentials in neonate rabbit SAN cells, which is useful to generate novel predictions regarding the key differences between the ion channel currents mechanism as contributors to the fast heart rate in neonatal cellular physiology.

\section{Acknowledgements}

This work was supported by King Abdul-Aziz University KAU, Jeddah, Saudi Arabia, and Saudi Cultural Bureau in the UK, London

\section{References}

1. Larson ED, St Clair JR, Sumner WA, Bannister RA, Proenza C. Depressed pacemaker activity of sinoatrial node myocytes contributes to the age-dependent decline in maximum heart rate. Proc Natl Acad Sci U S A [Internet]. 2013 Oct 29 [cited 2018 Mar 16];110(44):18011-6. Available from: http://www.ncbi.nlm.nih.gov/pubmed/24128759

2. Dobrzynski H, Boyett MR, Anderson RH. New Insights Into Pacemaker Activity: Promoting Understanding of Sick Sinus Syndrome. Circulation [Internet]. 2007 Mar 26 [cited 2018 Jan 5];115(14):1921-32. Available from: http://www.ncbi.nlm.nih.gov/pubmed/17420362

3. Zhang H, Holden A V, Kodama I, Honjo H, Lei M,
Varghese $\mathrm{T}$, et al. Mathematical models of action potentials in the periphery and center of the rabbit sinoatrial node. Am. J. Physiol. Heart Circ. Physiol. 279:H397-H421.

4. Baruscotti M, DiFrancesco D, Robinson RB. A TTXsensitive inward sodium current contributes to spontaneous activity in newborn rabbit sino-atrial node cells. J Physiol [Internet]. 1996 Apr 1 [cited 2018 Jan 30];492 ( $\mathrm{Pt}$ 1(Pt 1):21-30. Available from: http://www.ncbi.nlm.nih.gov/pubmed/8730579

5. Protas LE V, Difrancesco D, Robinson RB, Difrancesco D, Rob- RB. L-type but not T-type calcium current changes during postnatal development in rabbit sinoatrial node. 2017;10032:1252-9.

6. Accili EA, Robinson RB, DiFrancesco D. Properties and modulation of If in newborn versus adult cardiac SA node. Am J Physiol Circ Physiol [Internet]. 1997 Mar [cited 2018 Jan 30];272(3):H1549-52. Available from: http://www.physiology.org/doi/10.1152/ajpheart.1997. 272.3.H1549

7. Yang ZF, Sun Y, Li CZ, Wang HW, Wang XJ, Zheng $Y Q$, et al. Reduced sinoatrial cAMP content plays a role in postnatal heart rate slowing in the rabbit. Clin Exp Pharmacol Physiol. 2006;

8. Protas L, Oren R V., Clancy CE, Robinson RB. Agedependent changes in Na current magnitude and TTXsensitivity in the canine sinoatrial node. J Mol Cell Cardiol [Internet]. 2010 Jan [cited 2018 Mar 22];48(1):172-80. Available from: http://www.ncbi.nlm.nih.gov/pubmed/19665465

9. Honjo H, Boyett MR, Kodama I, Toyama J. Correlation between electrical activity and the size of rabbit sinoatrial node cells. J Physiol [Internet]. 1996 Nov 1 [cited 2018 Feb 20];496 ( Pt 3)(Pt 3):795-808. Available from: http://www.ncbi.nlm.nih.gov/pubmed/8930845

10. Allah EA, Tellez JO, Yanni J, Nelson T, Monfredi O, Boyett MR, et al. Changes in the expression of ion channels, connexins and $\mathrm{Ca}^{2+}$-handling proteins in the sino-atrial node during postnatal development. Exp Physiol [Internet]. 2011;96(4):426-38. Available from: http://doi.wiley.com/10.1113/expphysiol.2010.055780

11. Jones SA, Lancaster MK, Boyett MR. Ageing-related changes of connexins and conduction within the sinoatrial node. J Physiol. 2004;5602:429-37.

12. Irisawa H, Brown HF, Giles W. Cardiac pacemaking in the sinoatrial node. Physiol Rev [Internet]. 1993 Jan [cited 2018 Mar 12];73(1):197-227. Available from: http://www.ncbi.nlm.nih.gov/pubmed/8380502

Address for correspondence

Azzah M. Alghamdi

School of Physics and Astronomy, the University of

Manchester, Road,M139PL Oxford

(azzah.alghmdi@postgrad.manchester.ac.uk) 